\title{
The economic value of controlling for large losses in portfolio selection
}

\author{
Alexandra Dias* \\ School of Management \\ University of Leicester
}

\begin{abstract}
Research on asset pricing has shown that investor preferences include asymmetry and tail heaviness which affects the composition of optimal portfolios. This article investigates the out-of-sample economic value of introducing the risk of very large losses in portfolio selection. We combine mean-variance analysis with conditional Value-at-Risk using the subadditivity property of conditional Value-at-Risk, and we introduce a two stage method that preserves diversification while controlling for large losses. We find that strategies that account both for variance and the probability of large losses outperform efficient meanvariance portfolios, during and after the global financial crisis.
\end{abstract}

JEL classification: G11; G14

Keywords: portfolio selection, portfolio tail probability, conditional Value-at-Risk, risk management.

${ }^{*}$ Correspondence address: Alexandra Dias, School of Management University of Leicester, LE1 7RH Leicester, UK. Tel: +44(0)116 252 5019. E-mail: Alexandra.Dias@le.ac.uk 


\section{Introduction}

Large losses in financial markets are more frequent and larger than expected under the classical Markowitz (1952) framework. This is due to the non-normality of asset returns and has been recognized since Mandelbrot (1963). Portfolios composed using the classical "normal" mean-variance portfolio optimization are subject to potential large losses originated by the fat-tailedness of asset returns. Hence the need to incorporate the risk of large losses happening in portfolio selection.

It has been shown that investor preferences include asymmetry and tail heaviness; see Harvey and Siddique (2000), Dittmar (2002), Smith (2007), Guidolin and Timmermann (2008), Kozhan et al. (2013). Hence, introducing higher order moments in portfolio optimization affects the composition of optimal portfolios; see Guidolin and Timmermann (2008), Jondeau and Rockinger (2006, 2012).

In the existing literature one stream of research investigates the effect of including higher moments in portfolio selection. This requires the estimation of possibly many high-order crossmoments; see Martellini and Ziemann (2010). Another stream focuses on constraining the portfolio downside risk via Value-at-Risk (VaR), conditional Value-at-Risk (CVaR) or spectral risk measures; see Rockafellar and Uryasev (2000, 2002), Alexander and Baptista (2002), Adam et al. (2008), Brandtner (2013). This literature focuses mostly on probabilistic properties and estimation methods rather than on the economic significance of considering the possibility of large losses in the criteria for portfolio selection. Our contribution is to evaluate the economic value of two portfolio selection strategies that we propose combining mean-variance with the risk of large losses hapenning.

The concept of limiting downside risk goes back to Roy (1952) who introduced into portfolio selection the principle of safety-first. Roy used the two first moments of the asset returns distribution to limit the probability of a disastrous loss. The study of portfolio selection 
for safety-first investors was then based on the assumption of normally distributed asset returns. Later Arzac and Bawa (1977) introduced an essentially distribution free approach and used Value-at-Risk (VaR) as a downside risk measure. Another paper on portfolio allocation with safety-first without the normality assumption is Gourieroux et al. (2000) who use a nonparametric estimate of the full distribution of the asset returns. Jansen et al. (2000) concentrate on estimating the portfolio fat-tail distribution using the safety-first principle combined with extreme value theory to limit downside risk. More recently Jondeau and Rockinger (2006) introduce higher moments in portfolio selection and Adam et al. (2008), and Brandtner (2013) concentrate on using spectral measures of risk. Through time portfolio selection has departed from the assumption of normality.

Criteria for portfolio selection based on the tail properties of the asset returns distribution often choose a corner solution, meaning that most weight goes to the asset with the thinnest tail. This has been observed by, for instance, Jansen et al. (2000), Hartmann et al. (2004), Poon et al. (2003), Adam et al. (2008), and Brandtner (2013). The theoretical explanation for this is linked with a result from Geluk and de Haan (1987). They show that the tail-heaviness of the convolution of heavy-tailed variables is determined mainly by the variable with the heaviest tail. This means that the tail of a portfolio is mostly determined by the tail of the asset with the heaviest tail. As a consequence a portfolio strategy that minimizes tail risk leads to corner solutions by rejecting heavy tailed assets and allocating most weight to the asset with the lighter tail. Corner solutions are a serious drawback in the use of tail risk in portfolio selection because it discourages diversification. Recent work has been done exploring theoretical and empirical aspects of using tail risk measures such as spectral risk measures, a class which includes CVaR. Adam et al. (2008) find that risk measures that account primarily for worst case scenarios tend to overweight assets with thinner tails reducing diversification. Brandtner (2013) shows theoretically that portfolio selection using spectral risk measures tend to produce 
corner solutions. Brandtner and Kürsten (2014) show in the setting of optimal reinsurance that in particular using CVaR to find the optimal deductible also leads to corner solutions. For the case of optimal reinsurance Brandtner and Kürsten (2014) find that power spectral risk measures can overcome the corner solution problem. Hyung and de Vries (2007) also attempt to overcome the corner solution problem by using a second order expansion at infinity of the asymptotically Pareto tail probability. In our empirical study we take a simpler route. In our first approach we make use of the subadditivity property of the CVaR and obtain optimal mean-variance-CVaR portfolios. Our second approach consists of choosing the portfolio with the lowest CVaR among the set of all possible efficient mean-variance portfolios. With this two stage criterion, on the one hand we do not lose the diversification effect of mean-variance portfolios, and on the other hand we keep the probability of large losses under control essential during the non-normal "heavy-tailed" market times. With the second approach in the first stage we use mean-variance which ensures diversification and avoids corner solutions. In the second stage we keep the risk of large losses under control by selecting the efficient portfolio with the lowest tail risk.

In order to evaluate the economic value of the proposed strategies we consider an investor who takes into account the risk of large losses. Our investor likes mean and positive skewness, and dislikes variance and kurtosis; see Scott and Horvath (1980). Using a concept in the spirit of the certainty equivalent we estimate the fee that an investor would be willing to pay to move from a mean-variance strategy to each of the proposed alternative strategies. A positive fee means that the proposed strategy has a higher economic value for the investor than the mean-variance strategy.

The data used in our analysis consist of stock returns on ten industries covering the U.S. equity market. We choose to analyze a period of time before the global financial crisis, the period during the crisis, and the post-crisis. We compare our benchmark strategy, mean- 
variance, with the strategies involving CVaR, and we also include an equally weighted portfolio.

Our results indicate that the strategies that control for variance and CVaR outperform mean-variance, a mean-CVaR strategy and the equally weighted portfolio. The performance is measured by the economic value given by a mean-variance equivalent. We include the Sharpe ratio, and the Sortino ratio in the results for comparison. The results are most striking during the global financial crisis and after the crisis.

The organization of this paper is as follows. In Section 2 we describe the methodology used in our study. In Section 3 we present the empirical study and the results obtained. Section 4 concludes the paper.

\section{Methodology}

We implement the proposed strategies on the U.S. equity market using daily data available from the Kenneth R. French data library ${ }^{1}$. We choose to use the data where equities are grouped into ten industry portfolios spanning from January 1999 to December 2014. The industry portfolios are equally weighted portfolios. In the following we refer to industry portfolios or to assets interchangeably.

The benchmark strategy is the classical mean-variance Merton (1972) approach where the investor minimizes the variance of the portfolio for a given level of return. In our alternative strategies the investor prefers a high mean and a low variance, as in the mean-variance approach, and dislikes the risk of incurring large losses in the portfolio. We use as a measure of risk of incurring large losses the CVaR, also known as expected shortfall. Denoting by $R_{t}$ a random variable with a continuous distribution function representing the period $t$ return on an asset or industry portfolio, the CVaR at probability level $\alpha$ for period $t$ is given by the expected value

\footnotetext{
${ }^{1}$ Data downloaded from http://mba.tuck. dartmouth.edu/pages/faculty/ken.french/data_library.html.
} 
of the losses larger than the $100 \alpha \% \mathrm{VaR}$,

$$
\mathrm{CVaR}_{t}=-E\left(R_{t} \mid R_{t} \leq-100 \alpha \% \mathrm{VaR}_{t}\right)
$$

The VaR of probability level $\alpha$ is the quantile

$$
\left.\operatorname{VaR}_{t}=-\inf \left\{r \in \mathbb{R}: P\left(R_{t} \leq r \mid \mathcal{F}_{t-1}\right) \geq 1-\alpha\right)\right\}
$$

where $\mathcal{F}_{t}$ represents the information available at time $t$. The probability level $\alpha$ typically takes values between $90 \%$ and $99 \%$.

In line with related work, as Adam et al. (2008), Fleming et al. (2001), Jondeau and Rockinger (2006), we assume that short selling is not allowed in all the strategies in our study. This restriction makes our results more relevant for unsophisticated private investors or institutional investors who cannot use short selling, as for instance pension funds. Our goal here is to evaluate the effect of considering large losses on the performance of a portfolio of risky assets. Hence, we follow Jondeau and Rockinger (2006) and assume that the investor does not hold neither bonds nor cash in the portfolio.

The calculation of the portfolio weights for each strategy depends on the estimation of asset expected returns and $\mathrm{CVaR}$ based on historical data. For the estimation of tail risk measures (for instance in the area of risk measurement) 1,000 observations strike a balance between a sufficiently large number of observations for statistical estimation and a small enough time window for the estimates to be sensitive to changes in the market conditions. The Basel Committee (2013) favors the argument that the historical data used should reflect time varying market conditions and recommends to use one or two years of historical data but at the cost of larger errors. Hence, to increase the quality of the estimates we use 1,000 observations corresponding to four years of data. We use the window of the first 1,000 days of data to determine the portfolio weights for each strategy. The weights of the portfolio are then kept constant for one week and after one week we recalculate the portfolio weights according to the 
different strategies using the previous 1,000 days of data. We continue with this procedure for the different strategies until December 2014, obtaining 605 out-of-sample weekly returns for each strategy. We follow Jondeau and Rockinger (2006) in using a weekly portfolio optimization in our study. We do not use higher frequency for rebalancing the portfolio because in that case the gains from a better portfolio strategy might tend to disappear due to transaction costs.

\subsection{Portfolio allocation strategies}

We implement four portfolio optimization strategies. The first strategy is the mean-variance which is known for taking full advantage of the diversification effect. The second strategy is a mean-variance-CVaR strategy which constrains the portfolio tail risk. But as it is known that portfolio optimization considering worst case scenarios leads to corner solutions, Adam et al. (2008), we also implement a two stage mean-variance-CVaR where CVaR is only considered after mean-variance and not simultaneously as in the one stage mean-variance-CVaR strategy. We expect this nuance to make a difference especially during crisis periods. The fourth optimization strategy is the mean-CVaR which is used as a control strategy that optimizes the portfolio using only tail risk. For comparison we also include the equally-weighted portfolio corresponding to the $1 / N$ strategy studied in De Miguel et al. (2009). We give the details of each optimization strategy in the following.

\subsubsection{Mean-variance}

Our main benchmark strategy is the mean-variance strategy where the investor minimizes the portfolio variance for a given level of return $\bar{\mu}$. The optimization problem

$$
\min _{w} \frac{1}{2} w^{\prime} \Sigma w \quad \text { subject to } \quad w^{\prime} \boldsymbol{\mu}=\bar{\mu}, w^{\prime} \mathbf{1}=1, w_{i} \geq 0(i=1, \ldots, n)
$$

gives the vector of the optimal portfolio weights $w$ for the variance-covariance matrix $\Sigma$ and vector of expected asset returns $\boldsymbol{\mu}=E(\mathbf{R})$. 1 denotes the vector of ones with dimension $n$ 
corresponding to the number of assets.

To implement the mean-variance strategy we have to obtain a forecast of the variancecovariance matrix and asset returns. Fleming et al. (2001) showed that volatility timing has economic value in asset allocation. We follow Fleming et al. (2001) and use daily returns to forecast the conditional variance-covariance matrix $\Sigma$ as daily returns have a higher forecasting power than lower frequency returns. The matrix $\Sigma=\left[\sigma_{i j}\right]_{i, j=1, \ldots, 10}$ is obtained using the nonparametric one sided rolling estimator, studied in Foster and Nelson (1996) and used in Fleming et al. (2001), given by

$$
\hat{\sigma}_{i j, t}=\sum_{l=1}^{T} \omega_{i j, t-l}\left(r_{i, t-l}-\mu_{i}\right)\left(r_{j, t-l}-\mu_{j}\right),
$$

where $r_{i, t}$ and $r_{j, t}$ denote the returns on assets $i$ and $j$ respectively. $\omega_{i j, t}$ is the weight placed on the product of the return innovations for time $t . T$ is the number of observations in the sample window. Foster and Nelson (1996) show that the optimal weights are given by

$$
\omega_{i j, t-l}=\alpha_{i j, t} e^{-\alpha_{i j, t}|l|}
$$

where $\alpha_{i j, t}$ is the decay rate. We use $\alpha_{i j, t}=0.06$ following Fleming et al. (2001) and Foster and Nelson (1996).

In order to forecast the expected return $\boldsymbol{\mu}$ we could use a time series model. But typically due to large noise it is very difficult to forecast the average return; see Brandt (2009). Hence, we assume that the expected return $\boldsymbol{\mu}$ is constant in the estimation window and set at the same value over the incoming week when computing the portfolio weights. The data used spans over a period of 15 years including the 2007-2009 global financial crisis, pre-crisis, and post-crisis periods. Given that the market conditions changed during the sample period seems reasonable to assume that the investor's target return changed over time. We follow the methodology in Adam et al. (2008) and we assume that the investor's target return for period $t, \bar{\mu}$ (for all the strategies) corresponds to the equally weighted portfolio. As Adam et al. (2008) observe this 
corresponds to a region where efficient portfolios are significantly diversified. It also avoids choosing a level of expected return which is unattainable for an efficient portfolio.

\subsubsection{Mean-variance-CVaR}

With this strategy we assume that the investor's preferences are captured by the portfolio return, variance and risk of large losses measured by CVaR. In the general case, there is no closed form expression for the CVaR of a portfolio as a function of the individual assets' CVaR. This is because it depends on the dependence structure of the multivariate asset returns distribution. The direct minimization of portfolio CVaR was studied by Rockafellar and Uryasev (2000, 2002). Here we use the fact that CVaR is subadditive (Artzer et al., 1999) meaning that the portfolio CVaR is less or equal than the sum of the CVaR of the individual assets in the portfolio. Hence, the sum of the CVaR of the individual assets provides an upper bound for the portfolio CVaR.

Taking the subadditivity property of CVaR into consideration we define the asset allocation optimization problem as

$$
\min _{w} \frac{1}{2}\left(\beta w^{\prime} \Sigma w+(1-\beta) w^{\prime} \gamma\right) \quad \text { subject to } \quad w^{\prime} \boldsymbol{\mu}=\bar{\mu}, w^{\prime} \mathbf{1}=1, w_{i} \geq 0(i=1, \ldots, n)
$$

where $\gamma$ is the vector containing the CVaR for each asset, and $\beta \in(0,1]$.

When $\beta=1$ the optimization problem (4) corresponds to the mean-variance case. We performed the optimization for the cases of $\beta \in\{0.25,0.5,0.75\}$. We obtained better results for $\beta=0.5$. For reasons of space, we refrain from reporting the numerical results for $\beta \in$ $\{0.25,0.75\}$. For the probability level $\alpha$ of the CVaR, we consider and report three different cases $\alpha \in\{90 \%, 95 \%, 99 \%\}$, for each strategy and time period.

We note that this strategy does not take into account the possible tail diversification because the CVaR used is the sum of the individual assets' CVaR. We find interesting that although tail 
diversification is not considered by this strategy it still performs better, as we will see below in our empirical study, than one of the alternative strategies which takes tail diversification into account.

\subsubsection{Two stage mean-variance-CVaR}

In the calculation of the weights for the mean-variance-CVaR strategy as explained above there are two aspects playing a different role in the optimization. On the one hand, the minimization of the portfolio variance gives a considerable weight to several assets ensuring (variance) diversification. On the other hand, minimizing CVaR tends to lead to corner solutions due to the fact that the portfolio with the lowest tail heaviness contains mainly the asset with the thinnest tail. This happens because, as shown by Geluk and de Haan (1987), the notion of diversification is not valid for the tails of assets.

In order to keep the diversification effect but at the same time control for large losses we propose a simple two stage procedure. In the first stage we determine the portfolios that minimize the variance for a given target return considering all the possible combinations of assets. In our case as we have ten assets (industry sectors), there are 1,013 optimal meanvariance portfolios. In the second stage, from the 1,013 mean-variance optimal portfolios we choose the one with the lowest CVaR. We must note that this strategy takes into account the dependence structure of the tails of the asset returns (and possible tail diversification) while the single stage mean-variance-CVaR strategy does not.

\subsubsection{Mean-CVaR}

In order to facilitate a comparison with the mean-variance and mean-variance-CVaR strategies we also implement the mean-CVaR strategy. In this case the asset allocation optimization 
problem is

$$
\min _{w} \mathrm{CVaR} \quad \text { subject to } \quad w^{\prime} \boldsymbol{\mu}=\bar{\mu}, w^{\prime} \mathbf{1}=1, w_{i} \geq 0(i=1, \ldots, n) .
$$

We note that in this strategy there is tail diversification but no variance diversification.

\subsubsection{Estimation of CVaR}

The optimization strategies involving large losses imply the estimation of CVaR. We use the peaks-over-threshold (POT) method to estimate $\mathrm{CVaR}$ which is perhaps the most popular statistical method for estimating the tail heaviness of a random variable. For an application in finance see for instance Longin and Solnik (2001). The POT method is based on a theoretical result from Balkema and de Haan (1974) and Pickands (1975).

Let $R$ be a random variable denoting the portfolio returns with distribution function $F_{R}$. For simplicity we concentrate here on the tail of the positive values of the variable. Apart from a change of sign everything works analogously for the negative values.

The upper end point of the density function associated with $F_{R}$ is denoted by $x_{F}$. In the case of a normally distributed random variable, for instance, $x_{F}=+\infty$. The extremes of the variable are defined in terms of the exceedances over a threshold $u<x_{F}$. The distribution function of the random variable representing the exceedances of $R$ over the threshold $u$ is $F_{u}(x)=$ $P(R-u<x \mid R>u), x \geq 0$. The POT method uses the following result from extreme value theory that gives an approximation for the distribution function of the exceedances. Balkema and de Haan (1974) and Pickands (1975) show that there exists a unique non degenerate limit distribution, $G_{\xi, \beta}$, such that $\lim _{u \rightarrow x_{F}} \sup _{0<x<x_{F}-u}\left|F_{u}(x)-G_{\xi, \beta}(x)\right|=0 .^{2}$

The limit distribution $G_{\xi, \beta}$ is the Generalized Pareto distribution and has the form

$$
G_{\xi, \beta}(x)=1-(1+\xi x / \beta)^{-1 / \beta}
$$

\footnotetext{
${ }^{2}$ This result is also valid for non-i.i.d. processes; see Leadbetter et al. (1983).
} 
where $x \geq 0$ if $\xi \geq 0$, and $0 \leq x \leq-\beta / \xi$ if $\xi<0$. $\xi$ is the shape parameter and $\beta$ is a parameter depending on $u^{3}$. The probability level $\alpha$ CVaR is then given by

$$
\mathrm{CVaR}_{\alpha}=\frac{\operatorname{VaR}_{\alpha}}{1-\xi}+\frac{\beta-\xi u}{1-\xi}
$$

where the probability $\alpha$ VaR is

$$
\operatorname{VaR}_{\alpha}=u+\frac{\beta}{\xi}\left[\left(\frac{1-\alpha}{\bar{F}(u)}\right)^{-\xi}-1\right]
$$

$\bar{F}($.) denotes the survival distribution function of the variable $R$.

We estimate CVaR for period $t$ using the previous 1,000 observations. We estimate the parameters $\xi$ and $\beta$ of the Generalized Pareto distribution by maximum likelihood. More details about the statistical procedure can be found for instance in Embrechts et al. (1997). The survival distribution function is estimated using the empirical distribution function. The threshold $u$ is set such that $15 \%$ of the losses are larger than $u$. This is a value commonly used in the literature; for a recent application see Chavez-Demoulin et al. (2014) and references therein.

In order to challenge the parametric CVaR estimates we use the nonparametric estimator (see Adam et al. (2008) for further details)

$$
\mathrm{CVaR}_{\alpha}=-\frac{1}{n(1-\alpha)}\left(\sum_{i=1}^{\lceil n(1-\alpha)\rceil-1} x_{i: n}+(n(1-\alpha)-\lceil n(1-\alpha)\rceil+1) x_{\lceil n(1-\alpha)\rceil: n}\right),
$$

where in our case $\alpha \in\{0.9,0.95,0.99\}, x_{i: n}$ represent the ordered return observations, and $\lceil v\rceil$ is the smallest integer not less than $v$. We estimated the values of CVaR for the ten industry sectors in our data for every week from January 2003 to December 2014, at the three levels of confidence. The correlation between each series of parametric CVaR estimates and nonparametric CVaR estimates is above $99 \%$ in most cases and it is never below $94 \%$.

\footnotetext{
${ }^{3}$ Distributions with a power tail (heavy tailed distributions as the Student- $t$ for instance) have $\xi>0$, distributions with exponential tail (thin tailed distributions as the normal distribution) have $\xi=0$ and distributions with a finite tail (as the uniform distribution for instance) have $\xi<0$.
} 
The nonparametric estimator converges as the number of observations tends to infinity to the CVaR under the true distribution function. But, although the correlations between the estimates obtained from the two methods are very high, because we use an estimation window of 1,000 observations, which might be considered small for nonparametric estimation, we used the parametric estimates in our study.

\subsection{Measurement of the economic value}

To measure the value of incorporating the probability of large losses in portfolio selection we compare the ex-post performance of the strategies which incorporate the tail properties to that of the mean-variance strategy. If the inclusion of CVaR has no value the post performance of the mean-variance and the strategies including CVaR should be statistically the same. We need a measure of performance that encompasses the trade-off between risk and return. First we compute and report the Sharpe ratio. Although the Sharpe ratio only considers return and variance we decide to report it because it is widely used and hence it would be interesting to compare its values with other measures. As a second measure of performance we calculate the Sortino ratio which considers downside risk by replacing the variance in the Sharpe ratio with the negative semivariance. But Sharpe and Sortino ratios do not take into account the preferences of the investor relating the tail of the loss distribution. Hence, we use a third measure of performance that incorporates not only mean return and variance but also skewness and kurtosis. The last measure of performance is based on the concept of certainty equivalent and measures the economic value of each strategy in comparison with mean-variance. We define the mean-variance equivalent as the return from a mean-variance strategy that the investor would accept instead of an alternative strategy.

Scott and Horvath (1980) show that expected return and skewness have a positive contribution to the expected utility, while variance and kurtosis have a negative contribution. Also, 
Jondeau and Rockinger (2006) show that three- or four-moment optimization strategies provide a good approximation to the expected utility. We assume that the preferences of the investor are captured by a Taylor expansion of order four of a standard power utility function of terminal wealth $W_{t}$ with constant coefficient of relative risk aversion $\delta$. The investor likes high return and positive skewness, and dislikes variance and kurtosis. The Taylor series expansion of the utility function $U$ around the initial wealth $w_{t-1}$ is given by

$$
U\left(W_{t} ; \delta\right)=\sum_{n=0}^{m} \frac{1}{n !} U^{(n)}\left(w_{t-1} ; \delta\right)\left(W_{t}-w_{t-1}\right)^{n}+\epsilon_{m},
$$

where $U^{(0)}()=.U(),. U^{(n)}($.$) denotes the n$th derivative of the utility function with respect to final wealth $W_{t}$, and the remainder $\epsilon_{m}$ is of the order $o\left(\left(W_{t}-w_{t-1}\right)^{m}\right)$; see Tsiang (1972) for a study of the convergence conditions for a Taylor expanded power utility function. If we set $m=4$ then the expected utility is approximated by

$$
E\left[U\left(W_{t} ; \delta\right)\right] \approx \sum_{n=0}^{4} \kappa_{n} E\left[\left(W_{t}-w_{t-1}\right)^{n}\right],
$$

where $\kappa_{n}=\frac{1}{n !} U^{(n)}\left(w_{t-1} ; \delta\right)$. The expected utility depends on the first four moments of the distribution of the final wealth, where $\kappa_{1}$ and $\kappa_{3}$ are positive, and $\kappa_{2}$ and $\kappa_{4}$ are negative:

$$
\begin{aligned}
\kappa_{0} & =\frac{w_{t-1}^{1-\delta}}{1-\delta} \\
\kappa_{1} & =w_{t-1}^{-\delta} \\
\kappa_{2} & =-\frac{1}{2} \delta w_{t-1}^{-\delta-1} \\
\kappa_{3} & =\frac{1}{6} \delta(\delta+1) w_{t-1}^{-\delta-2}, \\
\kappa_{4} & =-\frac{1}{24} \delta(\delta+1)(\delta+2) w_{t-1}^{-\delta-3} .
\end{aligned}
$$

The (future) final wealth is given by $W_{t}=\left(1+R_{p, t}\right) w_{t-1}$, where the portfolio return $R_{p, t}$ (unknown at time $t-1$ ) is the product of the vector of individual asset returns by the vector of portfolio weights. Setting $w_{t-1}=1$ the realized utility at time $t$ is given by

$$
U\left(w_{t} ; \delta\right)=U\left(1+r_{p, t} ; \delta\right) \approx \sum_{n=0}^{4} \kappa_{n} r_{p, t}^{n} .
$$


As the coefficient of relative risk aversion is constant we can approximate the expected utility by the average realized utility

$$
\hat{E}\left[U\left(W_{t} ; \delta\right)\right] \approx \frac{1}{T} \sum_{j=0}^{T-1} U\left(1+r_{p, t-j} ; \delta\right)
$$

where in our empirical study $T=605$ weeks.

The value of a strategy is estimated by equating the average realized utilities for the two alternative strategies. We suppose that holding a portfolio other than the mean-variance portfolio subject to expenses $\Delta$ yields the same average utility as holding a mean-variance portfolio. An investor should be indifferent between these two strategies. Hence, $\Delta$ is the maximum fee that an investor would be willing to pay to switch from a mean-variance portfolio to the alternative strategy. If $\Delta$ is expressed as a fraction of the initial wealth invested, $\Delta$ solves the equation

$$
\sum_{j=0}^{T-1} U\left(1+r_{p, t-j} ; \delta\right)=\sum_{j=0}^{T-1} U\left(1+r_{s, t-j}-\Delta ; \delta\right),
$$

where $r_{p, t}$ and $r_{s, t}$ denote the returns on the mean-variance portfolio and on the alternative strategy portfolio, respectively. We report the results for $\delta=2, \delta=5$, and $\delta=10$.

An additional remark should be made here. In the estimation of the economic value of a strategy we follow Fleming et al. (2001) where the mean-variance equivalent is the return from a mean-variance strategy that the investor would equally prefer to the return on the alternative strategy. Hence, the return corresponding to the certainty equivalent in this setting, $r_{p, t}$ in Equation 8 , is time varying. But the value $\Delta$ which reveals if the alternative strategy would have higher economic value for the investor during the period considered is estimated as a constant for each period of time considered (pre-crisis, crisis, and post-crisis). 


\section{Data and empirical results}

The data collected from the Kenneth R. French library consists of daily stock returns covering the period from January 1999 to December 2014. Stocks are grouped into ten equally weighted industry portfolios. The ten industry portfolios are listed in Table 1. Sector ten labeled "Other" includes mines, construction, building maintenance, transportation, hotels, business services, entertainment, and finance. We use a 1,000 window of past data to calculate the weights for each strategy every week starting in January 2003 and spanning until December 2014. The summary statistics of the stock returns are reported in Table 1.

We split the period from January 2003 to December 2014 into three subperiods isolating the global financial crisis. We use the information from the National Bureau of Economic Research on U.S. Business Cycle Expansions and Contractions for the start and end dates of the global financial crisis. The subperiods considered in this study are: Pre-crises from January 2003 to November 2007; Crisis from December 2007 to June 2009; Post-crisis from July 2009 to December 2014. We perform the analysis and report the results for each of these subperiods as well as for the entire period.

The annualized realized mean returns are reported in Table 1 . We observe that the average return varies considerably across the different subperiods. During the crisis period six sectors have a negative mean return. The standard deviation shows an increase during the crisis period for all sectors. We also report the negative and positive semivariances. The negative semivariance is larger than the positive semivariance for all sectors before and after the crisis. During the crisis period four out the ten sectors have positive semivariance larger than negative semivariance. The realized frequency of negative returns is always below $50 \%$ except for three sectors during the crisis period. All sectors have negative skewness before and after the crisis. During the crisis half of the sectors have positive skewness. This result is perhaps counterintuitive. We would expect negative skewness to be more pronounced during the crisis 
Table 1: Summary statistics of returns on sectors

\begin{tabular}{|c|c|c|c|c|c|c|c|}
\hline & Mean & $\mathrm{SD}$ & $\mathrm{SD}^{-}$ & $\mathrm{SD}^{+}$ & $p^{-}$ & $m^{3}$ & $m^{4}$ \\
\hline \multicolumn{8}{|c|}{ Panel A: All sample 2003:01 - 2014:12 } \\
\hline Non Durables & 0.1967 & 0.1796 & 0.1908 & 0.1701 & 0.43 & -0.33 & 6.60 \\
\hline Durables & 0.1480 & 0.2496 & 0.2563 & 0.2435 & 0.46 & -0.07 & 6.57 \\
\hline Manufacturing & 0.2317 & 0.2347 & 0.2532 & 0.2198 & 0.43 & -0.31 & 6.76 \\
\hline Energy & 0.2090 & 0.3424 & 0.3635 & 0.3230 & 0.46 & -0.35 & 9.05 \\
\hline High Tech & 0.2372 & 0.2105 & 0.2227 & 0.2007 & 0.43 & -0.21 & 3.73 \\
\hline Telecommunications & 0.1924 & 0.2333 & 0.2438 & 0.2241 & 0.44 & -0.11 & 6.41 \\
\hline Shops & 0.2165 & 0.2071 & 0.2167 & 0.1989 & 0.44 & -0.21 & 6.16 \\
\hline Health & 0.2731 & 0.1929 & 0.2037 & 0.1845 & 0.44 & -0.36 & 4.53 \\
\hline Utilities & 0.1569 & 0.1708 & 0.1782 & 0.1646 & 0.44 & 0.28 & 15.7 \\
\hline Other & 0.1798 & 0.1877 & 0.1950 & 0.1817 & 0.44 & -0.08 & 7.67 \\
\hline \multicolumn{8}{|c|}{ Panel B: Pre-crisis period 2003:01 - 2007:11 } \\
\hline Non Durables & 0.1739 & 0.1032 & 0.1079 & 0.0997 & 0.43 & -0.42 & 1.35 \\
\hline Durables & 0.0992 & 0.1412 & 0.1459 & 0.1367 & 0.46 & -0.26 & 0.75 \\
\hline Manufacturing & 0.3054 & 0.1437 & 0.1527 & 0.1382 & 0.40 & -0.35 & 0.76 \\
\hline Energy & 0.4485 & 0.2166 & 0.2260 & 0.2106 & 0.42 & -0.35 & 0.58 \\
\hline High Tech & 0.2994 & 0.1657 & 0.1733 & 0.1608 & 0.41 & -0.21 & 0.35 \\
\hline Telecommunications & 0.1747 & 0.1584 & 0.1673 & 0.1511 & 0.43 & -0.23 & 0.88 \\
\hline Shops & 0.2402 & 0.1298 & 0.1365 & 0.1254 & 0.41 & -0.26 & 0.69 \\
\hline Health & 0.2755 & 0.1398 & 0.1437 & 0.1377 & 0.43 & -0.25 & 0.42 \\
\hline Utilities & 0.2106 & 0.1202 & 0.1286 & 0.1142 & 0.41 & -0.38 & 1.33 \\
\hline Other & 0.1977 & 0.1061 & 0.1091 & 0.1046 & 0.42 & -0.25 & 1.22 \\
\hline \multicolumn{8}{|c|}{ Panel C: Crisis period 2007:12 - 2009:06 } \\
\hline Non Durables & -0.0209 & 0.3383 & 0.3479 & 0.3277 & 0.47 & -0.01 & 1.17 \\
\hline Durables & -0.1265 & 0.4811 & 0.4618 & 0.5009 & 0.52 & 0.16 & 1.26 \\
\hline Manufacturing & -0.0781 & 0.4428 & 0.4564 & 0.4282 & 0.49 & -0.08 & 1.55 \\
\hline Energy & -0.0022 & 0.6559 & 0.6888 & 0.6217 & 0.47 & -0.12 & 2.67 \\
\hline High Tech & 0.0441 & 0.3484 & 0.3538 & 0.3427 & 0.47 & 0.04 & 1.37 \\
\hline Telecommunications & -0.0380 & 0.4316 & 0.4173 & 0.4457 & 0.52 & 0.14 & 1.74 \\
\hline Shops & 0.0772 & 0.3962 & 0.3910 & 0.4003 & 0.49 & -0.01 & 1.00 \\
\hline Health & 0.2167 & 0.3091 & 0.3107 & 0.3066 & 0.47 & -0.05 & 2.18 \\
\hline Utilities & -0.0688 & 0.3236 & 0.3333 & 0.3143 & 0.46 & 0.54 & 6.95 \\
\hline Other & 0.0041 & 0.3703 & 0.3566 & 0.3824 & 0.51 & 0.13 & 1.35 \\
\hline \multicolumn{8}{|c|}{ Panel D: Post-crisis period 2009:07 - 2014:12 } \\
\hline Non Durables & 0.2915 & 0.1670 & 0.1784 & 0.1585 & 0.42 & -0.57 & 4.04 \\
\hline Durables & 0.2959 & 0.2276 & 0.2396 & 0.2185 & 0.44 & -0.40 & 2.52 \\
\hline Manufacturing & 0.2742 & 0.2136 & 0.2282 & 0.2024 & 0.42 & -0.32 & 3.13 \\
\hline Energy & 0.0905 & 0.3020 & 0.3186 & 0.2857 & 0.47 & -0.47 & 3.48 \\
\hline High Tech & 0.2472 & 0.1935 & 0.2072 & 0.1821 & 0.43 & -0.43 & 2.83 \\
\hline Telecommunications & 0.2984 & 0.2073 & 0.2191 & 0.1983 & 0.43 & -0.42 & 3.34 \\
\hline Shops & 0.2400 & 0.1838 & 0.1924 & 0.1763 & 0.44 & -0.38 & 3.71 \\
\hline Health & 0.2901 & 0.1907 & 0.2072 & 0.1777 & 0.43 & -0.63 & 3.01 \\
\hline Utilities & 0.1819 & 0.1441 & 0.1459 & 0.1426 & 0.45 & -0.20 & 4.19 \\
\hline Other & 0.2214 & 0.1661 & 0.1763 & 0.1581 & 0.43 & -0.39 & 3.66 \\
\hline
\end{tabular}

Summary statistics of the daily price returns for each sector: annualized mean, standard deviation (SD), negative semivariance $\left(\mathrm{SD}^{-}\right)$, positive semivariance $\left(\mathrm{SD}^{+}\right)$, realized frequency of negative returns $\left(p^{-}\right)$, realized skewness $\left(m^{3}\right)$ and realized excess kurtosis $\left(m^{4}\right)$. Sector "Other" includes mines, construction, building maintenance, transportation, hotels, business services, entertainment, and finance. 
Table 2: Correlations in changes of conditional CVaR

\begin{tabular}{lccccccccc}
\hline & Non Durab & Durables & Manufact. & Energy & Hi Tech & Telecom & Shops & Health & Util \\
\hline Durables & 0.9048 & - & - & - & - & - & - & - & - \\
Manufact & 0.9057 & 0.8842 & - & - & - & - & - & - & - \\
Energy & 0.6332 & 0.6088 & 0.7081 & - & - & - & - & - & - \\
High Tech & 0.7303 & 0.7154 & 0.7357 & 0.5693 & - & - & - & - & - \\
Telecom & 0.8153 & 0.7887 & 0.7897 & 0.6106 & 0.8186 & - & - & - & - \\
Shops & 0.9326 & 0.9071 & 0.8997 & 0.6536 & 0.7881 & 0.8461 & - & - & - \\
Health & 0.7443 & 0.7389 & 0.7381 & 0.5776 & 0.8523 & 0.7786 & 0.7789 & - & - \\
Utilities & 0.7933 & 0.7427 & 0.7934 & 0.6777 & 0.6086 & 0.7781 & 0.7966 & 0.6208 & - \\
Other & 0.8914 & 0.8885 & 0.8777 & 0.6505 & 0.7321 & 0.8432 & 0.9115 & 0.7493 & 0.8006 \\
\hline
\end{tabular}

Correlations between the CVaR changes for the ten different sectors. The CVaR changes are positively strongly correlated across all sectors.

period. The results from the excess kurtosis are also interesting. The values of the kurtosis for the full period are higher than for each of the subperiods. This suggests that some type of structural change might have happened from period to period. This result is in line with results obtained supporting the existence of regimes switching; see for instance Guidolin and Timmermann (2008). Before the crisis the kurtosis takes its lowest values. The kurtosis increases during the crisis, especially for the utilities sector. After the crisis the values of kurtosis decrease but stay higher than they were before the crisis for all the sectors.

From the data we estimate the CVaR for the ten sectors during 605 weeks. As expected, the CVaR estimates change over time. We computed a time series of CVaR weekly changes for each sector and the correlation between the sectors CVaR changes. Table 2 has the results. The CVaR changes are strikingly highly correlated across all sectors.

\subsection{Portfolio results analysis}

We implement the four strategies mean-variance, mean-variance-CVaR, two stage mean-varianceCVaR, and mean-CVaR obtaining 605 realized portfolio returns. For comparison we also include the results for the equally weighted portfolio. The results are reported in Table 3. 


\subsubsection{Portfolio mean returns analysis}

Before the crisis the two stage mean-variance-CVaR strategy has the lowest annualized mean return while during and after the crisis it has the highest return, especially during the crisis. Before the crisis the mean-variance and the equally weighted portfolios have the highest mean returns. In the post-crisis period the two stage mean-variance-CVaR and equally weighted strategies have the highest and very close mean returns. During the crisis period the results are very different. Perhaps surprisingly the mean-CVaR strategy has the lowest return followed by the equally weighted and mean-variance strategies. The mean-variance CVaR has a higher return than the three previous strategies but it is the two stage mean-variance CVaR that remarkably outperforms all other strategies during the crisis. The fact that the mean-CVaR strategy has the lowest return during the crisis suggests that variance is still an important component of risk during unstable periods. In summary, the two stage mean-variance-CVaR produced the highest mean return during and after the financial crisis. Although the equally weighted portfolio has a high mean return before and after the crisis we will see that this return is also associated with the highest standard deviation.

\subsubsection{Sharpe ratio results}

The Sharpe ratio only considers variance as measure of risk but we report it for purposes of comparison. The riskless rate used to calculate the Sharpe ratio is the one-month USTreasury bond rate. In the pre-crisis period the mean-variance strategy has the highest Sharpe ratio. After the crisis the two stage mean-variance-CVaR has the highest Sharpe ratio closely followed by the mean-variance portfolio. During the crisis the mean-CVaR strategy has a negative Sharpe ratio. The mean-variance strategy also has a low Sharpe ratio although not as low as the equally weighted portfolio. The two stage mean-variance-CVaR has a Sharpe ratio remarkably higher than the other strategies during the crisis. Hence, both during and after the 
crisis the two stage mean-variance-CVaR has the highest Sharpe ratio.

\subsubsection{Sortino ratio results}

The Sortino ratio uses downside risk to measure the performance of the strategy. The conclusions are surprisingly similar to those obtained with the Sharpe ratio. During the crisis the strategies considering CVaR perform better, especially the two stage mean-variance-CVaR which still performs well after the crisis.

\subsubsection{The economic value of the different strategies}

The last three columns of Table 3 contain the results for the economic value of the three CVaR strategies and for the equally weighted portfolio measured using the notion of mean-variance equivalent as defined in Section 2.2. The values of $\Delta$ (see Equation 8) reported in Table 3 correspond to the fee that an investor would be willing to pay to change from the meanvariance strategy to one of the alternative strategies. The results are different for the three different periods considered.

Before the crisis an investor would not be willing to change from the mean-variance strategy to any of the alternative strategies including the equally weighted portfolio.

During the crisis period an investor would move to the mean-variance-CVaR especially the two stage mean-variance-CVaR. We must note that the investor would not move to the meanCVaR strategy which shows that considering variance is still important during crisis periods. The investor would not change either to the equally weighted portfolio.

After the crisis the results are different between a less risk averse investor, with relative risk aversion $\delta=2$ or $\delta=5$, and a more risk averse investor with $\delta=10$. After the crisis a less risk averse investor would change to the two stage mean-variance-CVaR. But the more risk averse investor would not change to any other strategy. This is evidence that the equity market did 
not revert to the pre-crisis conditions after the crisis period. In summary, the mean-varianceCVaR strategy outperformed the mean-variance strategy during the crisis period. The two stage mean-variance-CVaR strategy has been outperforming all other strategies since the crisis period.

\subsubsection{Further comments on the results}

We recall that the CVaR used in the one stage mean-variance-CVaR strategy is the sum of the individual assets $\mathrm{CVaR}$ which misses possible tail risk diversification. But although not taking into account the possible tail diversification this strategy still performs better, especially during the crisis period, than the mean-CVaR strategy which fully considers tail diversification.

We observe that the Sharpe ratio of the mean-CVaR strategy is not superior to the Sharpe ratio of the mean-variance strategy. We conclude that CVaR is not helping to predict other aspects of risk including variance. Theoretically this result makes sense. As shown by Brandtner and Kürsten (2014) and Acerbi (2004), spectral risk measures (CVaR is a spectral risk measure) are a linear combination of the expected value and another spectral measure where this last spectral measure captures only "pure" risk. CVaR is an example of such "pure" risk measure. This justifies our empirical observation where the mean-CVaR strategy does not have a higher Sharpe ratio than the mean-variance strategy.

\subsubsection{The probability level of CVaR}

The results reported in Table 3 consider a 95\% probability level CVaR. In order to investigate the sensitivity of the results to the level of probability used we performed also the calculations assuming the levels of $90 \%$ and $99 \%$. The results are listed in Table 6 in the Appendix. We observe that the results are coherent across the three levels of probability. Before the crisis there are not large differences with the strategies using $\alpha=90 \%$ performing slightly better. 
During the crisis the strategy using $95 \%$ performs better. After the crisis it is the $99 \%$ level mean-variance-CVaR, and the $95 \%$ two stage mean-variance-CVaR strategies that consistently have higher performance according to all measures.

\subsubsection{Summary statistics of the realized portfolio returns}

For completeness we report in Table 4 the summary statistics of the realized portfolio returns obtained with the different strategies. We remark here the most important results. During the pre-crisis period the mean-variance-CVaR strategies have the lowest standard deviation. During the crisis period the mean-variance-CVaR strategies have the higher return. The one stage mean-variance-CVaR has the lowest negative semivariance and the two stage mean-varianceCVaR has the smallest kurtosis. The mean-CVaR and the equally weighted portfolio have the largest kurtosis. After the crisis the mean-variance-CVaR together with the mean-variance strategy have the lowest standard deviation. The two stage mean-variance-CVaR has the largest mean return. The equally weighted portfolio has the largest standard deviation in all periods which is not surprising as this strategy assumes that the investor has no dislike for risk.

\subsection{Portfolio weights analysis}

In order to analyze the portfolio weights we consider the maximum portfolio weight in each of the 605 portfolios obtained using the four optimization strategies. We report in Table 5 the average maximum weights with the corresponding standard deviations. The results show that the mean-variance-CVaR strategies have the larger maximum weights except after the crisis. Safety-first strategies, based on minimizing the tail probability, are known to produce portfolios where most of the weight is in the stock with smaller loss probability (see for instance Jansen et al. (2000), Hartmann et al. (2004) and Poon et al. (2003)). That justifies why the mean-variance-CVaR strategy has maximum weight larger than the mean-variance strategy. 
Table 3: Ex-post portfolio performance

\begin{tabular}{|c|c|c|c|c|c|c|}
\hline & Mean & SR & SoR & $\Delta_{2}$ & $\Delta_{5}$ & $\Delta_{10}$ \\
\hline \multicolumn{7}{|c|}{ Panel A: All sample 2003:01 - 2014:12 } \\
\hline Mean-variance & 0.2106 & $\begin{array}{c}0.976 \\
{[0.421,1.622]}\end{array}$ & $\begin{array}{c}1.228 \\
{[0.506,2.362]}\end{array}$ & - & - & - \\
\hline Mean-var-CVaR & 0.1899 & $\begin{array}{c}0.880 \\
{[0.331,1.493]}\end{array}$ & $\begin{array}{c}1.136 \\
{[0.401,2.259]}\end{array}$ & $\begin{array}{c}-0.017 \\
{[-0.033,-0.001]}\end{array}$ & $\begin{array}{c}-0.017 \\
{[-0.033,-0.001]}\end{array}$ & $\begin{array}{c}-0.016 \\
{[-0.034,-0.000]}\end{array}$ \\
\hline Two-stage MVCVaR & 0.2019 & $\begin{array}{c}0.909 \\
{[0.329,1.583]}\end{array}$ & $\begin{array}{c}1.116 \\
{[0.392,2.214]}\end{array}$ & $\begin{array}{c}-0.009 \\
{[-0.046,0.031]}\end{array}$ & $\begin{array}{c}-0.013 \\
{[-0.048,0.025]}\end{array}$ & $\begin{array}{c}-0.021 \\
{[-0.056,0.015]}\end{array}$ \\
\hline Mean-CVaR & 0.1945 & $\begin{array}{c}0.841 \\
{[0.338,1.412]}\end{array}$ & $\begin{array}{c}1.134 \\
{[0.434,2.078]}\end{array}$ & $\begin{array}{c}-0.018 \\
{[-0.057,0.021]}\end{array}$ & $\begin{array}{c}-0.024 \\
{[-0.064,0.017]}\end{array}$ & $\begin{array}{c}-0.033 \\
{[-0.77,0.012]}\end{array}$ \\
\hline Equal weights & 0.2158 & $\begin{array}{c}0.813 \\
{[0.276,1.424]}\end{array}$ & $\begin{array}{c}1.060 \\
{[0.350,2.071]}\end{array}$ & $\begin{array}{c}-0.012 \\
{[-0.051,0.027]}\end{array}$ & $\begin{array}{c}-0.038 \\
{[-0.076,0.001]}\end{array}$ & $\begin{array}{c}-0.085 \\
{[-0.130,-0.040]}\end{array}$ \\
\hline \multicolumn{7}{|c|}{ Panel B: Pre-crisis period 2003:01 - 2007:11 } \\
\hline Mean-variance & 0.2385 & $\begin{array}{c}1.688 \\
{[0.804,2.637]}\end{array}$ & $\begin{array}{c}2.282 \\
{[1.297,4.978]}\end{array}$ & - & - & - \\
\hline Mean-var-CVaR & 0.2048 & $\begin{array}{c}1.447 \\
{[0.564,2.381]}\end{array}$ & $\begin{array}{c}2.590 \\
{[0.916,4.779]}\end{array}$ & $\begin{array}{c}-0.027 \\
{[-0.053,-0.001]}\end{array}$ & $\begin{array}{c}-0.026 \\
{[-0.054,-0.001]}\end{array}$ & $\begin{array}{c}-0.026 \\
{[-0.054,0.001]}\end{array}$ \\
\hline Two-stage MVCVaR & 0.1873 & $\begin{array}{c}1.309 \\
{[0.374,2.310]}\end{array}$ & $\begin{array}{c}2.078 \\
{[0.541,4.010]}\end{array}$ & $\begin{array}{c}-0.041 \\
{[-0.077,-0.006]}\end{array}$ & $\begin{array}{c}-0.041 \\
{[-0.080,-0.005]}\end{array}$ & $\begin{array}{c}-0.041 \\
{[-0.081,-0.003]}\end{array}$ \\
\hline Mean-CVaR & 0.2351 & $\begin{array}{c}1.439 \\
{[0.566,2.393]}\end{array}$ & $\begin{array}{c}2.157 \\
{[0.813,3.819]}\end{array}$ & $\begin{array}{c}-0.006 \\
{[-0.053,0.041]}\end{array}$ & {$[-0.013$} & $\begin{array}{c}-0.024 \\
{[-0.073,0.024]}\end{array}$ \\
\hline Equal weights & 0.2453 & $\begin{array}{c}1.368 \\
{[0.465,2.331]}\end{array}$ & $\begin{array}{c}2.160 \\
{[0.696,3.947]}\end{array}$ & $\begin{array}{c}-0.002 \\
{[-0.046,0.043]}\end{array}$ & $\begin{array}{c}-0.013 \\
{[-0.058,0.033]}\end{array}$ & $\begin{array}{c}-0.032 \\
{[-0.078,0.013]}\end{array}$ \\
\hline \multicolumn{7}{|c|}{ Panel C: Crisis period 2007:12 - 2009:06 } \\
\hline Mean-variance & 0.0261 & $\begin{array}{c}0.047 \\
{[-1.472,1.771]}\end{array}$ & $\begin{array}{c}0.069 \\
{[-2.104,3.137]}\end{array}$ & - & - & - \\
\hline Mean-var-CVaR & 0.0376 & $\begin{array}{c}0.079 \\
{[-1.505,1.835]}\end{array}$ & $\begin{array}{c}0.119 \\
{[-2.141,3.252]}\end{array}$ & $\begin{array}{c}0.010 \\
{[-0.031,0.056]}\end{array}$ & $\begin{array}{c}0.010 \\
{[-0.032,0.057]}\end{array}$ & $\begin{array}{c}0.009 \\
{[-0.035,0.059]}\end{array}$ \\
\hline Two-stage MVCVaR & 0.0752 & $\begin{array}{c}0.178 \\
{[-1.452,2.211]}\end{array}$ & $\begin{array}{c}0.254 \\
{[-2.058,3.515]}\end{array}$ & $\begin{array}{c}0.037 \\
{[-0.143,0.306]}\end{array}$ & $\begin{array}{c}0.020 \\
{[-0.157,0.267]}\end{array}$ & $\begin{array}{c}-0.014 \\
{[-0.170,0.211]}\end{array}$ \\
\hline Mean-CVaR & 0.0041 & $\begin{array}{c}-0.015 \\
{[-0.450,1.488]}\end{array}$ & $\begin{array}{c}-0.026 \\
{[-2.284,2.998]}\end{array}$ & $\begin{array}{c}-0.034 \\
{[-0.242,0.224]}\end{array}$ & $\begin{array}{c}-0.048 \\
{[-0.254,0.202]}\end{array}$ & $\begin{array}{c}-0.065 \\
{[-0.284,0.210]}\end{array}$ \\
\hline Equal weights & 0.0125 & $\begin{array}{c}0.006 \\
{[-1.484,1.723]}\end{array}$ & $\begin{array}{c}0.010 \\
{[-2.294,3.339]}\end{array}$ & $\begin{array}{c}-0.063 \\
{[-0.212,0.139]}\end{array}$ & $\begin{array}{c}-0.131 \\
{[-0.262,0.051]}\end{array}$ & $\begin{array}{c}-0.239 \\
{[-0.357,-0.062]}\end{array}$ \\
\hline \multicolumn{7}{|c|}{ Panel D: Post-crisis period 2009:07 - 2014:12 } \\
\hline Mean-variance & 0.2433 & $\begin{array}{c}1.278 \\
{[0.663,2.061]}\end{array}$ & $\begin{array}{c}1.615 \\
{[0.779,3.263]}\end{array}$ & - & - & - \\
\hline Mean-var-CVaR & 0.2235 & $\begin{array}{c}1.177 \\
{[0.588,1.904]}\end{array}$ & $\begin{array}{c}1.527 \\
{[0.713,3.029]}\end{array}$ & $\begin{array}{c}-0.016 \\
{[-0.038,0.005]}\end{array}$ & $\begin{array}{c}-0.016 \\
{[-0.039,-0.005]}\end{array}$ & $\begin{array}{c}-0.016 \\
{[-0.040,0.006]}\end{array}$ \\
\hline Two-stage MVCVaR & 0.2540 & $\begin{array}{c}1.281 \\
{[0.645,2.070]}\end{array}$ & $\begin{array}{c}1.583 \\
{[0.742,3.211]}\end{array}$ & $\begin{array}{c}0.006 \\
{[-0.036,0.054]}\end{array}$ & $\begin{array}{c}0.002 \\
{[-0.039,0.048]}\end{array}$ & $\begin{array}{c}-0.006 \\
{[-0.046,0.036]}\end{array}$ \\
\hline Mean-CVaR & 0.2179 & $\begin{array}{c}1.106 \\
{[0.480,1.842]}\end{array}$ & $\begin{array}{c}1.473 \\
{[0.616,2.824]}\end{array}$ & $\begin{array}{c}-0.023 \\
{[-0.060,0.015]}\end{array}$ & {$[-0.026$} & $\begin{array}{c}-0.030 \\
{[-0.071,0.010]}\end{array}$ \\
\hline Equal weights & 0.2536 & $\begin{array}{c}1.073 \\
{[0.442,1.843]}\end{array}$ & $\begin{array}{c}1.379 \\
{[0.537,2.790]}\end{array}$ & $\begin{array}{c}-0.007 \\
{[-0.059,0.050]}\end{array}$ & $\begin{array}{c}-0.031 \\
{[-0.085,0.025]}\end{array}$ & $\begin{array}{c}-0.076 \\
{[-0.136,-0.013]}\end{array}$ \\
\hline
\end{tabular}

Ex-post performance of five strategies: mean-variance, mean-variance-CVaR (Mean-var-CVaR), two stage meanvariance-CVaR (MVCVaR), mean-CVaR, and equally weighted (Equal weights). The ex-post performance is reported for the entire sample, the period before the sub-prime crisis, during the crisis, and after the crisis. For each case we report the annualized realized mean return (Mean), the realized Sharpe ratio (SR), and the realized Sortino ratio (SoR). $\Delta_{\delta}$ is the annualized percentage fee that an investor with constant risk aversion $\delta$ would be willing to pay to change from the mean-variance strategy to other strategy considered. The values in square parenthesis are $90 \%$ confidence bands estimated using block bootstrap with 50,000 replicates. 
Table 4: Summary statistics of the realized portfolio returns

\begin{tabular}{|c|c|c|c|c|c|c|c|}
\hline & Mean & SD & $\sigma^{-}$ & $\sigma^{+}$ & $p^{-}$ & $m^{3}$ & $m^{4}$ \\
\hline \multicolumn{8}{|c|}{ Panel A: All sample 2003:01 - 2014:12 } \\
\hline Mean-variance & 0.2106 & 0.1826 & 0.1941 & 0.1776 & 0.39 & -0.40 & 6.92 \\
\hline Mean-var-CVaR & 0.1899 & 0.1830 & 0.1925 & 0.1786 & 0.41 & -0.32 & 6.79 \\
\hline Two-stage MVCVaR & 0.2017 & 0.1880 & 0.2024 & 0.1803 & 0.40 & -0.60 & 6.65 \\
\hline Mean-CVaR & 0.1945 & 0.1959 & 0.2043 & 0.1924 & 0.41 & 0.02 & 5.89 \\
\hline Equal weights & 0.2158 & 0.2246 & 0.2385 & 0.2172 & 0.40 & -0.17 & 6.24 \\
\hline \multicolumn{8}{|c|}{ Panel B: Pre-crisis period 2003:01 - 2007:11 } \\
\hline Mean-variance & 0.2385 & 0.1102 & 0.1013 & 0.1219 & 0.41 & -0.25 & -0.07 \\
\hline Mean-var-CVaR & 0.2048 & 0.1095 & 0.0999 & 0.1204 & 0.42 & -0.16 & -0.16 \\
\hline Two-stage MVCVaR & 0.1873 & 0.1098 & 0.1054 & 0.1167 & 0.41 & -0.31 & -0.01 \\
\hline Mean-CVaR & 0.2351 & 0.1274 & 0.1292 & 0.1313 & 0.39 & -0.46 & 0.05 \\
\hline Equal weights & 0.2453 & 0.1401 & 0.1390 & 0.1458 & 0.40 & -0.33 & -0.08 \\
\hline \multicolumn{8}{|c|}{ Panel C: Crisis period 2007:12 - 2009:06 } \\
\hline Mean-variance & 0.0261 & 0.3410 & 0.3511 & 0.3283 & 0.46 & 0.11 & 1.28 \\
\hline Mean-var-CVaR & 0.0376 & 0.3420 & 0.3443 & 0.3359 & 0.47 & 0.13 & 1.25 \\
\hline Two-stage MVCVaR & 0.0752 & 0.3528 & 0.3642 & 0.3384 & 0.47 & -0.15 & 0.77 \\
\hline Mean-CVaR & 0.0041 & 0.3642 & 0.3481 & 0.3743 & 0.48 & 0.52 & 1.48 \\
\hline Equal weights & 0.0125 & 0.4128 & 0.4205 & 0.4017 & 0.44 & 0.33 & 1.45 \\
\hline \multicolumn{8}{|c|}{ Panel D: Post-crisis period 2009:07 - 2014:12 } \\
\hline Mean-variance & 0.2433 & 0.1703 & 0.1863 & 0.1637 & 0.38 & -0.88 & 5.6 \\
\hline Mean-var-CVaR & 0.2235 & 0.1712 & 0.1875 & 0.1636 & 0.38 & -0.82 & 5.29 \\
\hline Two-stage MVCVaR & 0.2540 & 0.1766 & 0.1947 & 0.1689 & 0.38 & -0.98 & 6.45 \\
\hline Mean-CVaR & 0.2179 & 0.1781 & 0.1906 & 0.1723 & 0.40 & -0.67 & 2.98 \\
\hline Equal weights & 0.2536 & 0.2105 & 0.2290 & 0.2009 & 0.39 & -0.77 & 4.13 \\
\hline
\end{tabular}

Summary statistics of the realized weekly portfolio returns: annualized mean, standard deviation (SD), negative semivariance $\left(\mathrm{SD}^{-}\right)$, positive semivariance $\left(\mathrm{SD}^{+}\right)$, realized frequency of negative returns $\left(p^{-}\right)$, skewness $\left(m^{3}\right)$, and excess kurtosis $\left(m^{4}\right)$. 
But these differences are only pronounced before the crisis. During and after the crisis the CVaR strategies do not overweight one asset when compared with mean-variance.

A possible feature of strategies that consider downside risk is high turnover. This is undesirable because it increases transaction costs consuming the gains obtained from considering CVaR besides variance.

Denote the portfolio weights at the beginning of week $t$, after choosing the new portfolio, by the $n \times 1$ vector $w_{t}$ and the realized asset returns on week $t$ by the $n \times 1$ vector $r_{t}$, where $n$ is the number of assets in the portfolio. The weight of asset $i$ in the portfolio during week $t$ is $w_{i, t}$ and the realized return on asset $i$ on week $t$ is $r_{i, t}$. The portfolio return in week $t$ is $r_{t} w_{t}$. The turnover rate in week $t$, after choosing the new portfolio, is

$$
T O_{t}=\sum_{i=1}^{n}\left|w_{i, t+1}-w_{i, t} \frac{1+r_{i, t}}{1+r_{t} w_{t}}\right| .
$$

In Table 5 we report the average turnover rate for the four strategies. We observe that before and after the crisis the mean-variance strategy has the highest turnover while the meanCVaR has the lowest. During the crisis the two stage mean-variance-CVaR strategy has the highest turnover while the mean-CVaR has the lowest. This result shows that the higher return during the crisis obtained by the two stage mean-variance-CVaR strategy is obtained with a higher turnover while the other strategies with a lower turnover have a much smaller return, especially the mean-CVaR which has the lowest return during the crisis. The results suggest that in order to keep a higher return during the crisis it is important to use a mean-varianceCVaR strategy and to keep the frequency of rebalancing the portfolio low. For completeness we add the corresponding results for the levels of confidence $90 \%$ and $99 \%$ in Table 7 in the Appendix. The results are consistent with the results for $95 \%$ discussed in this section. 
Table 5: Portfolio allocation analysis

\begin{tabular}{|c|c|c|c|}
\hline & \multirow[t]{2}{*}{ Turnover } & \multicolumn{2}{|c|}{ Maximum weight } \\
\hline & & Mean & $\mathrm{SD}$ \\
\hline \multicolumn{4}{|c|}{ Panel A: All sample 2003:01 - 2014:12 } \\
\hline Mean-variance & 0.333 & 0.584 & 0.145 \\
\hline Mean-var-CVaR & 0.198 & 0.675 & 0.160 \\
\hline Two-stage MVCVaR & 0.267 & 0.700 & 0.177 \\
\hline Mean-CVaR & 0.088 & 0.637 & 0.098 \\
\hline \multicolumn{4}{|c|}{ Panel B: Pre-crisis period 2003:01 - 2007:11 } \\
\hline Mean-variance & 0.359 & 0.607 & 0.153 \\
\hline Mean-var-CVaR & 0.219 & 0.744 & 0.172 \\
\hline Two-stage MVCVaR & 0.276 & 0.803 & 0.180 \\
\hline Mean-CVaR & 0.109 & 0.631 & 0.123 \\
\hline \multicolumn{4}{|c|}{ Panel C: Crisis period 2007:12 - 2009:06 } \\
\hline Mean-variance & 0.292 & 0.637 & 0.170 \\
\hline Mean-var-CVaR & 0.285 & 0.646 & 0.175 \\
\hline Two-stage MVCVaR & 0.451 & 0.649 & 0.190 \\
\hline Mean-CVaR & 0.007 & 0.630 & 0.000 \\
\hline \multicolumn{4}{|c|}{ Panel D: Post-crisis period 2009:07 - 2014:12 } \\
\hline Mean-variance & 0.324 & 0.547 & 0.119 \\
\hline Mean-var-CVaR & 0.150 & 0.621 & 0.117 \\
\hline Two-stage MVCVaR & 0.211 & 0.622 & 0.113 \\
\hline Mean-CVaR & 0.093 & 0.644 & 0.086 \\
\hline
\end{tabular}

This table illustrates the average turnover and the distribution of the asset weights for each portfolio strategy. We present the mean and the standard deviation of the maximum asset weights. SD stands for standard deviation.

\section{Conclusion}

The mean-variance approach to portfolio selection does not take into account the large losses that are observed in the market. Even when constraining the portfolio selection by minimizing the downside risk, if multivariate normality is assumed, the potential portfolio large losses are still most likely underestimated. To overcome this shortcoming researchers have included higher moments in portfolio selection, or estimated portfolio large losses using extreme risk measures. The first approach quickly becomes unfeasible because a large number of cross-moments has to be estimated; the second approach usually yields corner solutions where most of the portfolio weight is on the asset with the thinnest tail. With some exceptions, the literature in this area has left largely unanswered the question of the economic value of taking large losses into account in portfolio selection.

Our study shows that mean-variance portfolios with lower probability of portfolio large 
losses, measure by CVAR, outperform mean-variance portfolios especially during the last global financial crisis. We find that with this methodology on the one hand we improve the performance of mean-variance and on the other hand we avoid the corner solutions of extreme risk measures only strategies. We find that an investor is willing to pay a positive fee to change from the mean-variance to a mean-variance-CVaR strategy where there is a lower probability of large losses.

Using a two stage procedure the mean-variance-CVaR strategy leads to a significantly better ex-post performance when compared with a one stage optimization procedure. This result is explained by the fact that mean-variance implies diversification but minimizing portfolio tail probabilities reduces the diversification effect by allocating most of the wealth to the asset with the thinnest tail.

Considering CVaR could lead to strategies with higher turnover rates associated with higher transaction costs. But our study shows that this only happens during the crisis period and for the two stage mean-variance-CVaR strategy. Otherwise mean-variance has the highest turnover. Overall we observe that during the crisis both mean-variance-CVaR and two stage mean-variance-CVaR perform better than mean-variance, an equally weighted portfolio or a mean-CVaR strategy. This shows that considering CVaR during the crisis period is important but so it is to consider variance. After the crisis, the two stage mean-variance-CVaR keeps performing the best even for more risk averse investors.

The immediate implication of the results obtained in this study for the finance industry, namely banks and insurance companies, when building risky portfolios is that we should consider a measure of tail risk but variance is not to be disposed outright. A second possible implication, to be explored in further work, is that from the risk management point of view perhaps we should also include variance when measuring risk. Recall that now VaR is the measure of risk more often used by industry. 
It has been shown that the economic value of volatility timing can be improved by increasing the quality of the volatility estimation; see Fleming et al. (2003). We used the parametric peaksover-threshold method to estimate the CVaR and we found no significant difference between the parametric and a nonparametric estimator for CVaR. But it would be interesting in future work to focus on the possibility of improving the economic value of the mean-variance-CVaR strategy using more sophisticated measures of the portfolio tail risk. 


\section{References}

Acerbi, C. (2004). Coherent representations of subjective risk aversion. In: Risk Measures for the 21st Century (Ed. Giorgio Szegö), Wiley, Chichester, pages 147-208.

Adam, A., Houkari, M., and Laurent, J. P. (2008). Spectral risk measures and portfolio selection. Journal of Banking and Finance, 32:1870-1882.

Alexander, G. J. and Baptista, A. M. (2002). Economic implications of using a mean-VaR model for portfolio selection: a comparison with mean-variance analysis. Journal of Economic Dynamics \& Control, 26:1159-1193.

Artzer, P., Delbaen, F., Eber, J. M., and Heath, D. (1999). Coherent measures of risk. Mathematical Finance, 3:203-228.

Arzac, E. R. and Bawa, V. S. (1977). Portfolio choice and equilibrium in capital markets with safety-first investors. Journal of Financial Economics, 4:277-288.

Balkema, A. A. and de Haan, L. (1974). Residual life time at great age. The Annals of Probability, 2:792-804.

Basel Committee (2013). Fundamental review of the trading book: A revised market risk framework. Basel Committee on Banking Supervision, October 2013, Available from www . bis . org.

Brandt, M. (2009). Portfolio choice problems. In: Handbook of Financial Econometrics, vol 1 (Ed. L P Hansen and Y Aït-Sahalia), Amsterdam: North-Holland, pages 267-336.

Brandtner, M. (2013). Conditional Value-at-Risk, spectral risk measures and (non)diversification in portfolio selection problems - A comparison with mean-variance analysis. Journal of Banking and Finance, 37:5526-5537.

Brandtner, M. and Kürsten, W. (2014). Solvency II, regulatory capital, and optimal reinsurance: How good are Conditional Value-at-Risk and spectral risk measures? Insurance: Mathematics and Economics, 59:156-167.

Chavez-Demoulin, V., Embrechts, P., and Sardy, S. (2014). Extreme-quantile tracking for financial time series. Forthcoming in Journal of Econometrics, 181(1):44-52.

De Miguel, V., Garlappi, L., and Uppal, R. (2009). Optimal versus naive diversification: How inefficient is the 1/n portfolio strategy? The Review of Financial Studies, 22(5):1915-1953.

Dittmar, R. (2002). Non linear pricing kernels, kurtosis preference, and evidence fron the cross section of equity returns. Journal of Finance, 57:369-403.

Embrechts, P., Klüppelberg, C., and Mikosch, T. (1997). Modelling Extremal Events for Insurance and Finance. Springer-Verlag, Berlin.

Fleming, J., Kirby, C., and Ostdiek, B. (2001). The economic value of volatility timing. Journal of Finance, LVI(1):329-351.

Fleming, J., Kirby, C., and Ostdiek, B. (2003). The economic value of volatility timing using "realized" volatility. Journal of Financial Economics, 67:473-509.

Foster, D. P. and Nelson, D. B. (1996). Continuous record asymptotics for rolling sample variance estimators. Econometrica, 64:139-174. 
Geluk, J. and de Haan, L. (1987). Regular variation, extensions and Tauberian theorems. CWI Tract, vol. 40, CWI, Amsterdam.

Gourieroux, C., Laurent, J. P., and Scaillet, O. (2000). Sensitivity analysis of values at risk. Journal of Empirical Finance, 7:225-246.

Guidolin, M. and Timmermann, A. (2008). International asset allocation under egime switching, skewness and kurtosis preferences. Review of Financial Studies, 21:889-935.

Hartmann, P., Straetmans, S., and de Vries, C. G. (2004). Asset market linkages in crisis periods. Review of Economics and Statistics, 86:313-326.

Harvey, C. and Siddique, A. (2000). Conditional skewness in asset pricing tests. Journal of Finance, 55:1263-1295.

Hyung, N. and de Vries, C. G. (2007). Portfolio selection with heavy tails. Journal of Empirical Finance, 14:383-400.

Jansen, D., Koedijk, K. J., and de Vries, C. G. (2000). Portfolio selection with limited down-side risk. Journal of Empirical Finance, 7:247-269.

Jondeau, E. and Rockinger, M. (2006). Optimal portfolio allocation under high moments. European Financial Management, 12:29-55.

Jondeau, E. and Rockinger, M. (2012). On the importance of time variability in higher moments for asset allocation. Journal of Empirical Econometrics, 10:84-123.

Kozhan, R., Neuberger, A., and Schneider, P. (2013). The skew risk premium in the equity index market. Review of Financial Studies, 26:2174-2203.

Leadbetter, M. R., Lindgren, G., and Rootzén, H. (1983). Extremes and Related Properties of Random Sequences and Processes. Springer Verlag, New York.

Longin, F. and Solnik, B. (2001). Extreme correlation of international equity markets. Journal of Finance, LVI(2):649-676.

Mandelbrot, B. B. (1963). The variation of certain speculative prices. Journal of Business, $36: 394-419$.

Markowitz, H. M. (1952). Portfolio selection. Journal of Finance, 7:77-91.

Martellini, L. and Ziemann, V. (2010). Improved estimates of higher-order comoments and implications for portfolio selection. Review of Financial Studies, 23(4):1467-1502.

Merton, R. C. (1972). An analytic derivation of the efficient portfolio frontier. Journal of Financial and Quantitative Analysis, 7:1851-1872.

Pickands, J. (1975). Statistical inference using extreme order statistics. The Annals of Statistics, $3: 119-31$.

Poon, S. H., Rockinger, M., and Tawn, J. (2003). Nonparametric extreme value dependence measures and finance aplications. Statistica Sinica, 13:929-953.

Rockafellar, R. T. and Uryasev, S. (2000). Optimization of conditional Value-at-Risk. Journal of Risk, 2:21-41. 
Rockafellar, R. T. and Uryasev, S. (2002). Conditional Value-at-Risk for general loss distributions. Journal of Banking and Finance, 26:1443-1471.

Roy, A. D. (1952). Safety first and holding of assets. Econometrica, 20:431-49.

Scott, R. C. and Horvath, P. A. (1980). On the direction of preference for moments of higher order than variance. Journal of Finance, 35:915-919.

Smith, D. R. (2007). Conditional coskewness and asset pricing. Journal of Empirical Finance, 14:91-119.

Tsiang, S. C. (1972). The rationale of the mean-standard deviation analysis, skewness preference, and the demand for money. American Economic Review, 62:75-111. 
5 Appendix 
Table 6: Ex-post portfolio performance for different confidence levels CVaR

\begin{tabular}{|c|c|c|c|c|c|c|}
\hline & Mean & SR & SoR & $\Delta_{2}$ & $\Delta_{5}$ & $\Delta_{10}$ \\
\hline \multicolumn{7}{|c|}{ Panel A: All sample 2003:01 - 2014:12 } \\
\hline \multirow[t]{2}{*}{ Mean-var-CVaR 90\% } & 0.1891 & 0.877 & 1.128 & -0.017 & -0.017 & -0.017 \\
\hline & & {$[0.332,1.499]$} & {$[0.406,2.228]$} & {$[-0.034,-0.001]$} & {$[-0.034,-0.001]$} & {$[-0.034,-0.000]$} \\
\hline \multirow[t]{2}{*}{ Mean-var-CVaR 99\% } & 0.2000 & 0.903 & 1.188 & -0.010 & -0.013 & -0.017 \\
\hline & & {$[0.353,1.525]$} & {$[0.437,2.318]$} & {$[-0.038,0.019]$} & {$[-0.041,0.015]$} & {$[-0.045,0.011]$} \\
\hline \multirow[t]{2}{*}{ 2-st. MVCVaR $90 \%$} & 0.1836 & 0.843 & 1.054 & -0.023 & -0.024 & -0.026 \\
\hline & & {$[0.280,1.489]$} & {$[0.337,2.087]$} & {$[-0.057,0.014]$} & {$[-0.059,0.012]$} & {$[-0.063,0.011]$} \\
\hline \multirow[t]{2}{*}{ 2-st. MVCVaR 99\% } & 0.2008 & 0.888 & 1.113 & -0.011 & -0.017 & -0.028 \\
\hline & & {$[0.281,1.583]$} & {$[0.333,2.273]$} & {$[-0.057,0.035]$} & {$[-0.062,0.027]$} & {$[-0.072,0.018]$} \\
\hline \multirow{2}{*}{ Mean-CVaR $90 \%$} & 0.1927 & 0.847 & 1.140 & -0.018 & & -0.028 \\
\hline & & {$[0.347,1.412]$} & {$[0.446,2.085]$} & {$[-0.054,0.020]$} & {$[-0.06]$} & {$[-0.072,0.018]$} \\
\hline \multirow[t]{2}{*}{ Mean-CVaR $99 \%$} & 0.1908 & 0.821 & 1.122 & -0.021 & -0 & -0.037 \\
\hline & & {$[0.337,1.365]$} & {$[0.448,2.023]$} & {$[-0.061,0.020]$} & {$[-0.069$} & {$[-0.08$} \\
\hline \multicolumn{7}{|c|}{ Panel B: Pre-crisis period 2003:01 - 2007:11 } \\
\hline \multirow[t]{2}{*}{ Mean-var-CVaR $90 \%$} & 0.2101 & 1.493 & 2.651 & -0.022 & & -0.021 \\
\hline & & {$[0.590,2.435]$} & {$[0.988,4.869]$} & {$[-0.050,0.002]$} & {$[-0.05$} & {$[-0.05$} \\
\hline \multirow[t]{2}{*}{ Mean-var-CVaR $99 \%$} & 0.1725 & 1.164 & 2.127 & -0.054 & & -0.055 \\
\hline & & {$[0.278,2.101]$} & {$[0.485,4.180]$} & {$[-0.085,-0.023]$} & {$[-0.085$} & {$[-0.088,-0.023]$} \\
\hline \multirow[t]{2}{*}{ 2-st. MVCVaR $90 \%$} & 0.1905 & 1.335 & 2.133 & -0.038 & -0.038 & -0.038 \\
\hline & & {$[0.379,2.355]$} & {$[0.573,4.120]$} & {$[-0.076,-0.003]$} & {$[-0.076$} & {$[-0.078,-0.000]$} \\
\hline \multirow[t]{2}{*}{ 2-st. MVCVaR $99 \%$} & 0.2048 & 1.408 & 2.435 & -0.027 & & \\
\hline & & {$[0.433,2.445]$} & {$[0.696,4.788]$} & {$[-0.074$} & {$[-0.07$} & {$[-0.07$} \\
\hline \multirow[t]{2}{*}{ Mean-CVaR $90 \%$} & 0.2362 & 1.472 & 2.211 & -0.005 & -0 . & -0 . \\
\hline & & {$[0.597,2.431]$} & {$[0.868,3.881]$} & {$[-0.048,0.038]$} & {$[-0.05$} & {$[-0.06$} \\
\hline \multirow[t]{2}{*}{ Mean-CVaR 99\% } & 0.2463 & 1.456 & 2.158 & 0.000 & & -0 \\
\hline & & {$[0.612,2.401]$} & {$[0.880,3.803]$} & {$[-0.049,0.052]$} & {$[-0.05$} & {$[-0.07$} \\
\hline \multicolumn{7}{|c|}{ Panel C: Crisis period 2007:12 - 2009:06 } \\
\hline \multirow[t]{2}{*}{ Mean-var-CVaR $90 \%$} & 0.0313 & 0.061 & 0.092 & 0.004 & & \\
\hline & & {$[-1.473,1.801]$} & {$[-2.170,3.230]$} & {$[-0.041$} & {$[-0$} & {$[-0.0$} \\
\hline \multirow[t]{2}{*}{ Mean-var-CVaR $99 \%$} & 0.0125 & 0.008 & 0.012 & -0.0132 & -0 & -0.009 \\
\hline & & {$[-1.567,1.732]$} & {$[-2.288,3.158]$} & {$[-0.117,0.092]$} & {$[-0.12$} & {$[-0.12$} \\
\hline \multirow[t]{2}{*}{ 2-st. MVCVaR $90 \%$} & 0.0267 & 0.047 & 0.068 & -0.006 & -0 & -0.042 \\
\hline & & {$[-1.591,2.009]$} & {$[-2.227,3.191]$} & {$[-0.191,0.2$} & {$[-0.19$} & {$[-0.208,0.181]$} \\
\hline \multirow[t]{2}{*}{ 2-st. MVCVaR $99 \%$} & 0.0101 & 0.001 & 0.001 & -0.016 & -0 . & -0.023 \\
\hline & & {$[-1.768,2.143]$} & {$[-2.509,3.563]$} & {$[-0.244,0.269]$} & {$[-0.23$} & {$[-0.23$} \\
\hline Mean-CVaR $90 \%$ & -0.0006 & -0.028 & -0.048 & -0.035 & -0.0 & -0.051 \\
\hline & & {$[-1.420,1.4$} & {$[-2.208,2.7$} & {$[-0.237,0.2$} & {$[-0.249$} & {$[-0.278,0.235]$} \\
\hline Mean-CVaR 99\% & 0.0036 & -0.016 & -0.028 & -0.035 & -0 & -0.065 \\
\hline & & {$[-1.361,1.404]$} & {$[-2.140,2.821]$} & {$[-0.245,0.242]$} & {$[-0.26$} & {$[-0.302,0.245]$} \\
\hline & & Panel D: Post & risis period 20 & $9: 07-2014: 12$ & & \\
\hline Mean-var-CVaR 90\% & 0.2192 & 1.160 & 1.501 & -0.019 & & \\
\hline & & {$[0.577,1.876]$} & {$[0.705,3.003]$} & {$[-0.042,0.0$} & {$[-0.042,0.0$} & {$[-0.043,0.003]$} \\
\hline Mean-var-CVaR 99\% & 0.2862 & 1.397 & 1.810 & 0.031 & 0.025 & 0.016 \\
\hline & & {$[0.755,2.195]$} & {$[0.908,3.5$} & {$[-0.011,0.08$} & {$\left[-0.015,0.0^{\prime}\right.$} & {$[-0.021,0.059]$} \\
\hline 2-st. MVCVaR $90 \%$ & 0.2258 & 1.193 & 1.548 & -0.014 & -0.013 & -0.011 \\
\hline & & {$[0.618,1.896]$} & {$[0.763,2.918]$} & {$[-0.047,0.020]$} & {$[-0.049,0.022]$} & {$[-0.050,0.028]$} \\
\hline 2-st. MVCVaR $99 \%$ & 0.2566 & 1.215 & 1.508 & 0.004 & -0.007 & -0.028 \\
\hline & & {$[0.535,2.060]$} & {$[0.636,3.156]$} & {$[-0.044,0.05$} & {$[-0.056,0.046]$} & {$[-0.083,0.027]$} \\
\hline Mean-CVaR 90\% & 0.2146 & 1.106 & 1.479 & -0.024 & -0.026 & -0.028 \\
\hline & & {$[0.489,1.832]$} & {$[0.619,2.819]$} & {$[-0.059,0.011]$} & {$[-0.063,0.009]$} & {$[-0.067,0.011]$} \\
\hline Mean-CVaR 99\% & 0.2000 & 1.028 & 1.394 & -0.037 & -0.039 & -0.042 \\
\hline & & {$[0.408,1.741]$} & {$[0.528,2.720]$} & {$[-0.074,0.000]$} & {$[-0.077,-0.001]$} & {$[-0.084,0.001]$} \\
\hline
\end{tabular}

Ex-post performance of three strategies: mean-variance-CVaR (Mean-var-CVaR), two stage mean-varianceCVaR (MVCVaR), and mean-CVaR. We report the performance of these three strategies for three confidence levels of CVaR: $90 \%$, and 99\%. The ex-post performance is reported for the entire sample, the period before the sub-prime crisis, during the crisis, and after the crisis. For each case we report the annualized realized mean return (Mean), the realized Sharpe ratio (SR), and the realized Sortino ratio (SoR). $\Delta_{\delta}$ is the annualized percentage fee that an investor with constant risk aversion $\delta$ would be willing to pay to change from the meanvariance strategy to other strategy considered. The values in square parenthesis are $90 \%$ confidence bands estimated using block bootstrap with 50,000 replicates. 
Table 7: Portfolio allocation analysis

\begin{tabular}{|c|c|c|c|}
\hline & \multirow[t]{2}{*}{ Turnover } & \multicolumn{2}{|c|}{ Maximum weight } \\
\hline & & Mean & SD \\
\hline \multicolumn{4}{|c|}{ Panel A: All sample 2003:01 - 2014:12 } \\
\hline Mean-var-CVaR $90 \%$ & 0.193 & 0.677 & 0.156 \\
\hline Mean-var-CVaR $99 \%$ & 0.255 & 0.728 & 0.172 \\
\hline Two-stage MVCVaR 90\% & 0.239 & 0.680 & 0.174 \\
\hline Two-stage MVCVaR $99 \%$ & 0.294 & 0.648 & 0.157 \\
\hline Mean-CVaR 90\% & 0.071 & 0.626 & 0.094 \\
\hline Mean-CVaR $99 \%$ & 0.085 & 0.632 & 0.165 \\
\hline \multicolumn{4}{|c|}{ Panel B: Pre-crisis period 2003:01 - 2007:11 } \\
\hline Mean-var-CVaR $90 \%$ & 0.213 & 0.746 & 0.166 \\
\hline Mean-var-CVaR $99 \%$ & 0.185 & 0.801 & 0.147 \\
\hline Two-stage MVCVaR $90 \%$ & 0.264 & 0.756 & 0.197 \\
\hline Two-stage MVCVaR 99\% & 0.265 & 0.720 & 0.164 \\
\hline Mean-CVaR $90 \%$ & 0.091 & 0.599 & 0.105 \\
\hline Mean-CVaR $99 \%$ & 0.107 & 0.517 & 0.137 \\
\hline \multicolumn{4}{|c|}{ Panel C: Crisis period 2007:12 - 2009:06 } \\
\hline Mean-var-CVaR $90 \%$ & 0.310 & 0.634 & 0.176 \\
\hline Mean-var-CVaR $99 \%$ & 0.275 & 0.633 & 0.171 \\
\hline Two-stage MVCVaR 90\% & 0.406 & 0.662 & 0.183 \\
\hline Two-stage MVCVaR 99\% & 0.623 & 0.608 & 0.170 \\
\hline Mean-CVaR $90 \%$ & 0.008 & 0.648 & 0.000 \\
\hline Mean-CVaR 99\% & 0.006 & 0.796 & 0.000 \\
\hline \multicolumn{4}{|c|}{ Panel D: Post-crisis period 2009:07 - 2014:12 } \\
\hline Mean-var-CVaR $90 \%$ & 0.137 & 0.627 & 0.111 \\
\hline Mean-var-CVaR $99 \%$ & 0.313 & 0.689 & 0.168 \\
\hline Two-stage MVCVaR $90 \%$ & 0.171 & 0.616 & 0.111 \\
\hline Two-stage MVCVaR $99 \%$ & 0.231 & 0.594 & 0.115 \\
\hline Mean-CVaR $90 \%$ & 0.072 & 0.644 & 0.091 \\
\hline Mean-CVaR $99 \%$ & 0.089 & 0.688 & 0.140 \\
\hline
\end{tabular}

This table illustrates the average turnover and the distribution of the asset weights for each portfolio strategy. We present the mean and the standard deviation of the maximum asset weights. SD stands for standard deviation. 